\title{
Role of genetic polymorphisms and mutations in colorectal cancer therapy (Review)
}

\author{
MARCO AIELLO $^{1}$, NADIA VELLA ${ }^{1}$, CALOGERO CANNAVÒ $^{1}$, AURORA SCALISI ${ }^{2}$, DEMETRIOS A. SPANDIDOS ${ }^{5}$, \\ GIUSEPPE TOFFOLI $^{3}$, ANGELA BUONADONNA ${ }^{4}$, MASSIMO LIBRA ${ }^{1}$ and FRANCA STIVALA ${ }^{1}$ \\ ${ }^{1}$ Department of Biomedical Sciences, University of Catania; ${ }^{2}$ Secondary Prevention and Screening Gynaecological Unit, \\ AUSL 3, Catania; ${ }^{3}$ Experimental and Clinical Pharmacology; ${ }^{4}$ Medical Oncology Division B, Centro di Riferimento \\ Oncologico, IRCCS, Aviano, Italy; ${ }^{5}$ Department of Virology, Medical School, University of Crete, Heraklion, Greece
}

Received November 18, 2010; Accepted December 12, 2010

DOI: $10.3892 / \mathrm{mmr} .2010 .408$

\begin{abstract}
Gene polymorphisms and mutations in various types of cancer may predict clinical response to chemotherapy and related toxicity, since they may affect the metabolism of the drugs commonly used in combination chemotherapy treatments. However, conflicting data have been generated on this subject. To elucidate this issue, this review discusses the clinical applications of several genetic polymorphisms in colorectal cancer patients treated with the most common agents alone or in combination. UDP-glucuronosyltransferase (UGT)1A1 is a conjugating biotransformation enzyme that plays a role in maintaining the levels of endogenous compounds (e.g., bilirubin) and in handling exogenous compounds, including carcinogens. It has been demonstrated that the UGT1A1*28 polymorphism plays a predictive role in patients administered an irinotecancontaining regimen. Polymorphisms in XPD (Lys751Gln), a member of the nucleotide excision repair pathway, negatively affect response to therapy, with oxaliplatin/5FU reducing the survival of the patient. A similar reaction has also been observed in patients with the XRCC1 Arg399Gln polymorphism, while patients with the GSTP1 Ile105Val polymorphism have an improved response to oxaliplatin/5FU therapy. Treatment with biological compounds such as cetuximab, an anti-epidermal growth factor receptor (EGFR) monoclonal antibody, has been shown to be effective only in colon cancer patients with wild-type K-Ras. Fc polymorphisms are associated with progression-free survival in patients treated with cetuximab. Another monoclonal antibody useful in the treatment of colon cancer is bevacizumab, a monoclonal antibody to vascular endothelial growth factor (VEGF); however, in some cases bevacizumab may cause deep vein thrombosis (DVP). In a related vein, our recent unpublished data show that the VEGF
\end{abstract}

Correspondence to: Dr Massimo Libra, Department of Biomedical Sciences, University of Catania, Via Androne 83, 95124 Catania, Italy

E-mail: mlibra@unict.it

Key words: colorectal cancer, gene polymorphisms, biological therapy
C936T polymorphism may increase the risk of DVP in cancer patients. In conclusion, this review indicates that certain polymorphisms increase the effectiveness of certain drugs, while others greatly enhance their toxicity. The study of the genetic 'habitus' therefore appears to be crucial for the development of tailored therapy for cancer patients.

\section{Contents}

1. Introduction

2. Current therapy for colorectal cancer

3. Genetic alterations

4. Conclusion

\section{Introduction}

Colorectal cancer is the third most common cancer among males $(663,000$ cases, $10 \%$ of the total) and the second most common cancer among females (570,000 cases, 9.4\% of the total) worldwide. Approximately $60 \%$ of cases present in developed countries. There is a variation of 10 -fold in the incidence rates for both genders worldwide: Australia/New Zealand and Western Europe present the highest rates, Africa (excepting Southern Africa) and South-Central Asia have the lowest rates, while rates are intermediate in Latin America. In all areas, incidence rates are higher in men than women (overall gender ratio of the age-standardized incidence rates, 1.4:1).

Colorectal cancer is responsible of approximately 608,000 deaths worldwide ( $8 \%$ of all cancer-related deaths), making it the fourth most common cause of cancer-related death. As with the incidence rates, mortality rates are higher among males than females, with the exception of the Caribbean. However, mortality rates are less variable worldwide (6-fold for males, 5-fold for females): the highest mortality rates for both genders are found in Central and Eastern Europe (20.3 per 100,000 for males, 12.1 per 100,000 for females), while the lowest are in Middle Africa (3.5 and 2.7 per 100,000, respectively) (http:// globocan.iarc.fr/factsheets/cancers/colorectal.asp). In Italy, the incidence has risen from 16,000 cases in 1970 to over 37,000 cases in 2004. 
Risk factors for colorectal cancer include increased age, gender (men are more predisposed to the development of colorectal cancer), the presence of inflammatory bowel disease, certain hereditary conditions and a family history of colorectal cancer. It is unlikely that these risk factors will change. However, it should be noted that even in the absence of predisposing factors, the general population has an average risk of developing the disease, and approximately $75 \%$ of all cases of colorectal cancer occur in individuals with no known predisposing factors for the disease (1).

The incidence of colorectal cancer increases with age, with many elderly patients ( $65 \%)$ developing advanced disease.

\section{Current therapy for colorectal cancer}

Primary prevention of colorectal cancer is based on the implementation of screening tests to detect and remove pre-cancerous lesions or to discover and treat cancer at its earliest stages. The survival rate of patients with colorectal cancer depends on the clinical and pathological stage of the disease at diagnosis. Patients with cancer limited to the bowel wall at diagnosis have a 5-year survival rate of $90 \%$ (2). The survival rate is reduced to $35-60 \%$ if the lymph nodes are involved, and drops to less than $10 \%$ when the disease is metastatic (3) (http://www.ncbi.nlm. nih.gov/bookshelf/br.fcgi?book=techrev1\&part=A65).

There is full agreement among health care professionals regarding the essential role of surgery in the treatment of colorectal cancer. Further improvements are obtained through the use of treatments combining surgery, chemotherapy and radiotherapy, and in particular adjuvant and neoadjuvant therapies.

Various combinations of chemotherapeutic drugs are currently employed. 5FU, which was the first chemotherapeutic agent used to treat colorectal cancer, is still used today and is considered preferable to the FOLFIRI (folinic acid, FU and irinotecan) and FOLFOX (folinic acid, FU and oxaliplatin) protocols.

New biological compounds, such as bevacizumab, cetuximab and panitumumab, are also used in the treatment of colorectal cancer. In particular, vascular endothelial growth factor (VEGF) has proven to be the most powerful angiogenic factor. VEGF binds to its receptor, activing several intracellular signal transduction pathways (4). In addition, VEGF receptor- 2 was expressed on the surface of colorectal cancer cells in approximately $50 \%$ of samples analyzed (5). The role of VEGF in cancer progression is well known: VEGF and other pro-angiogenic factors stimulate the degradation of the extracellular matrix, leading to the proliferation and migration of endothelial cells (6). For this reason, both VEGF and VEGF receptors are valuable therapeutic targets.

Bevacizumab is a humanized monoclonal antibody against VEGF. It binds to VEGF, thereby preventing VEGF from binding to its receptor and halting the subsequent activation of intracellular signal transduction pathways. Previously, it was thought that high microvessel density and VEGF expression represented negative prognostic markers in patients with metastatic colorectal cancer $(7,8)$. However, it now appears that the response to bevacizumab is independent of VEGF expression or high microvessel density (9). Predictive markers of response to bevacizumab have not been identified to date.
The epidermal growth factor receptor (EGFR) is a member of the ErbB transmembrane TK receptor family. Various ligands (EGF, TGF- $\alpha$, epiregulin and amphiregulin) bind to this receptor and stimulate several intracellular signal transduction pathways (Ras/Raf/MAPK, PI3K/Akt and JAK-STAT), leading to cell proliferation, dedifferentiation and the arrest of apoptosis (10). In addition, EGFR has been observed to be expressed on the surface of colorectal cancer cells in approximately $40-70 \%$ cases, and has been associated with a decrease in survival (11-13).

Currently, two new anti-EGFR molecules are used to treat colorectal cancer: the monoclonal antibodies cetuximab and panitumumab. Cetuximab is a chimeric IgG1 antibody and panitumumab is a total human $\operatorname{IgG} 2$ antibody. Notably, it was determined that cetuximab can only be used in the absence of K-Ras oncogene mutations (14-18). K-Ras mutations are observed in approximately $38 \%$ of colorectal cancer patients (19), and cause the constitutive activation of the Ras/Raf/ MAPK pathway, which is not linked to EGFR activation by ligand binding (20). Since patients without K-Ras wild-type cancer cannot benefit from treatment with anti-EGFR antibodies, the European Medicines Agency has approved the use of these antibodies only in patients with K-Ras wild-type tumors (21).

Among patients with K-Ras wild-type tumors, anti-EGFR therapy appears to be further limited to patients with B-Raf wild-type tumors (22), although a recent study found that the B-Raf mutation was a prognostic rather than a predictive marker (23). Anti-VEGF and anti-EGFR antibodies have a proven efficacy. At present, bevacizumab, cetuximab and panitumumab are the standard therapeutic options for metastatic colorectal cancer.

Bevacizumab is administered in combination with chemotherapy as a first-line treatment, while anti-EGFR antibodies appear to be more beneficial in later-line treatment. Furthermore, anti-VEGF and anti-EGFR antibodies, in combination with chemotherapeutic agents, increased the life expectancy of patients with metastatic colorectal cancer by approximately 2 years.

The combination of anti-VEGF and anti-EGFR therapy has been proposed, since VEGF has many intracellular pathways in common with EGFR, and because colorectal cancer cells and endothelial cells express both EGFR and VEGF receptor-2 $(24,25)$. Although pre-clinical studies demonstrated that EGFR ligands up-regulated VEGF expression (26) and that the combination of antiangiogenic and anti-EGFR agents might have significant antitumor activity (27-30), numerous clinical trials have found that not only is the combination of the two antibodies less effective than the individual agents combined with other chemotherapeutic drugs, but also that their combination results in a reduction of progression-free and overall survival medians (31).

Currently, bevacizumab is commonly used in combination with fluoropyrimidine-based chemotherapy in the treatment of metastatic colorectal cancer. However, as yet no data is available from randomized clinical trials on the optimal duration of treatment with bevacizumab or other targeted agents.

The efficacy of anti-EGFR antibodies in the treatment of metastatic colorectal cancer is limited to patients with K-Ras wild-type cancer. However, it is undoubtedly necessary to 
Table I. Genetic alterations associated with current colon cancer therapies.

\begin{tabular}{|c|c|c|c|c|}
\hline Polymorphism & Effect of mutation & Anticancer agent(s) & Effect & Refs. \\
\hline $\begin{array}{l}\text { XPD Lys } 751 \mathrm{Gln} \\
\text { heterozygosis and } \\
\text { homozygosis }\end{array}$ & $\begin{array}{l}\text { Influence of DNA repair } \\
\text { capacity }\end{array}$ & Oxaliplatin/5FU & $\begin{array}{l}\text { Worse response, shorter } \\
\text { survival }\end{array}$ & $(36,37)$ \\
\hline UGT1A1*28 & $\begin{array}{l}\text { Reduced glucuronidation of } \\
\text { SN38 }\end{array}$ & Irinotecan & $\begin{array}{l}\text { Predictive role in response to an } \\
\text { irinotecan-containing regimen }\end{array}$ & (38) \\
\hline $\begin{array}{l}\text { GSTP1 Ile105Val } \\
\text { homozygosis }\end{array}$ & $\begin{array}{l}\text { Reduction of enzymatic } \\
\text { activity }\end{array}$ & Oxaliplatin/5FU & Longer survival & (39) \\
\hline $\begin{array}{l}\text { XRCC1 Arg399Gln } \\
\text { heterozygosis and } \\
\text { homozygosis }\end{array}$ & $\begin{array}{l}\text { Deficiency in DNA repair } \\
\text { pathways }\end{array}$ & Oxaliplatin/5FU & Shorter survival & $(40)$ \\
\hline $\begin{array}{l}\text { Fc- } \gamma \text { RIIa } 131 \mathrm{H} / \mathrm{H} / \\
\text { Fc- } \gamma \text { RIIIa } 158 \mathrm{~V} / \mathrm{V}\end{array}$ & $\begin{array}{l}\text { Higher affinity to human } \mathrm{IgG} 2 / \\
\text { higher affinity to human IgG1 }\end{array}$ & Cetuximab & $\begin{array}{l}\text { Longer progression-free } \\
\text { survival }\end{array}$ & $(32,41)$ \\
\hline Mutation of K-Ras & $\begin{array}{l}\text { Constitutive activation of the } \\
\text { Ras/Raf/MAPK pathway }\end{array}$ & Cetuximab & $\begin{array}{l}\text { No response to cetuximab-based } \\
\text { therapy }\end{array}$ & $(20)$ \\
\hline $\begin{array}{l}\text { Thymidylate synthase } \\
* 3 / * 3 \text { genotype }\end{array}$ & $\begin{array}{l}\text { Reduction of enzymatic } \\
\text { activity }\end{array}$ & $5 \mathrm{FU}$ & $\begin{array}{l}\text { Better response to 5FU- } \\
\text { based chemotherapy }\end{array}$ & $(42,43)$ \\
\hline MGMT $2535 \mathrm{G}>\mathrm{T}$ & $\begin{array}{l}\text { Reduction of enzymatic } \\
\text { activity }\end{array}$ & Oxaliplatin & Longer progression-free survival & $(45)$ \\
\hline $\begin{array}{l}\text { MTHFR } 677 \mathrm{C} / \mathrm{T} \\
\text { MTHFR } 1298 \mathrm{~A} / \mathrm{C}\end{array}$ & $\begin{array}{l}\text { Altered enzymatic activity } \\
\text { and hyperhomocysteinemia }\end{array}$ & FOLFOX regimen & $\begin{array}{l}\text { Longer time to progression and } \\
\text { overall survival }\end{array}$ & $(46-49)$ \\
\hline
\end{tabular}

further study other possible predictive markers of response to anti-EGFR therapy, such as B-Raf, PI3K and polymorphisms of the receptor gene Fc- $\gamma(23,31,32)$.

Over the past two decades, the role of the genetic pathways encoding the enzymes involved in metabolism, cellular transport and the mechanisms of action of chemotherapeutic agents have been investigated. Pharmacogenetic studies of cancer therapeutics have shown an association between specific genetic variants of drug metabolizing enzymes (pharmacogenetic determinants of response) and adverse drug reactions or toxicity. Primarily, it has been observed that genetic abnormalities influence response to treatment (33-35). In this review, the correlation between current colon cancer therapies and the genetic polymorphisms responsible for an increased response rate or lack of response to treatment and increased toxicity are described (Table I).

\section{Genetic alterations}

XPD Lys751Gln polymorphism and oxaliplatin/5FU. XPD (Xeroderma pigmentosum group D) is a member of the nucleotide excision repair (NER) family and plays an important role in the repair of DNA damage caused by alkylating drugs, platinum analogues and radiation. Protein levels of XPD were demonstrated to be correlated with resistance to these agents in human tumor cell lines (36). Moreover, a clinical trial on 73 metastatic colorectal cancer patients treated with a combination of 5FU and oxaliplatin demonstrated that patients with the Lys/Lys genotype at codon 751 responded better $(p=0.015)$ and had a longer survival $(\mathrm{p}=0.002)$ than patients with at least one mutated allele (37).

UGT1A1*28 polymorphism and CPT11. In a study of advanced colorectal cancer patients, Toffoli et al reported a stronger predictive role of the UGT1A $1{ }^{*} 28$ polymorphism in terms of response to an irinotecan-containing regimen, compared to its predictive role in determining drug-related toxicity, which was significant after the first cycle of chemotherapy, but less relevant over the entire course of treatment (38). This suggests the need for careful consideration before irinotecan dose reduction in patients carrying the UGT1A1*28 polymorphism.

GSTP1 Ile105Val polymorphism and oxaliplatin/5FU. The GSTP1 Ile105Val polymorphism was found to be associated with increased survival in patients with advanced colorectal cancer treated with oxaliplatin/5FU (39).

XRCC1 Arg399Gln polymorphism and oxaliplatin/5FU. In 61 colorectal cancer patients treated with oxaliplatin/5FU, the XRCC1 Arg399Gln polymorphism was linked to shorter survival $(\mathrm{p}=0.038)$, probably since this polymorphism is responsible for the emergence of resistance to this combination (40).

Germline Fc- $\gamma$ RIIa 131H/H and/or Fc- $\gamma$ RIIIa 158V/V polymorphisms and anti-EGFR agents. Germline polymorphisms of $\mathrm{Fc}-\gamma$ receptors have also been associated with response to anti-EGFR agents, with longer progression-free survival in 
patients that present the Fc- $\gamma$ RIIa $131 \mathrm{H} / \mathrm{H}$ and/or Fc- $\gamma$ RIIIa $158 \mathrm{~V} / \mathrm{V}$ polymorphism $(32,41)$.

Thymidylate synthase germline polymorphisms and $5 F U$. $5 \mathrm{FU}$ is the most commonly used drug in combined pre-operative chemotherapy (CT) and radiotherapy (RT) as standard treatment for colorectal cancer. 5FU is a folate pathway inhibitor. This fluoropyrimidine, activated in its nucleotide form, forms a stable complex with thymidylate synthase (TS), inhibiting enzyme function. TS catalyzes an important reaction for cell proliferation (reductive methylation of dUMP by 5,10-methylenetetrahydrofolate to form dTMP; Danenberg, 1977). The efficacy of 5FU is inversely proportional to TS activity. Our previous study demonstrated increased TS expression in liver metastases from colorectal cancer patients resistant to fluoropyrimidine-based chemotherapy (42).

Various polymorphisms in the gene encoding TS have been described. The most important genetic alteration of the TS gene consists of a variation in the number of the 28-bp tandemly repeated sequence, located in the 50-UTR region (43). Alleles with two $\left(\mathrm{TS}^{*} 2\right)$ and three $\left(\mathrm{TS}^{*} 3\right)$ repeats are the most common.

Pàez et al investigated the role of the TS genotype as a predictive marker in patients with locally advanced rectal cancer treated with a CT/RT regimen. They found that the TS *3/*3 genotype predicted the colorectal cancer patients who better responded to CT/RT 5FU-based treatment. The study of the TS genotype could therefore be useful to reveal patients with stage II/III colorectal cancer who could benefit from preand postoperative fluorouracil-based chemotherapy (44).

MGMT 2535G $>T$ polymorphism and oxaliplatin. O6-methylguanine-DNA methyltransferase is an enzyme necessary for the repair of DNA lesions induced by alkylating agents. It is involved in the DNA direct reversal repair pathway coping with alkylating agents, which is one of the underlying mechanisms of individual resistance to the chemotherapeutic effects of certain alkylating agents as well as carcinogenesis.

Park et al investigated 16 DNA repair gene polymorphisms in patients with advanced colorectal cancer treated with oxaliplatin-based therapy. In particular, they found that the MGMT $-535 \mathrm{G}>\mathrm{T}$ (rs1625649) polymorphism has a predictive effect on the survival of these patients. This genetical alteration is linked with the progression-free survival in patients with advanced colorectal cancer treated with oxaliplatin-based chemotherapy. This underlines the potential important role of DNA repair gene polymorphisms in predicting the clinical outcome of chemotherapy in metastatic colorectal cancer (45).

Methylenetetrahydrofolate reductase gene polymorphisms and FOLFOX chemotherapy. Methylenetetrahydrofolate reductase (MTHFR) is an enzyme that catalyzes the conversion of 5,10-methylenetetrahydrofolate $(\mathrm{CH} 2 \mathrm{FH} 4)$ to 5-methyltetrahydrofolate $(\mathrm{CH} 3 \mathrm{FH} 4)$, a co-substrate for homocysteine remethylation to methionine. Genetic variation in this gene is responsible for susceptibility to certain diseases, including colon cancer and acute leukemia.

The two most common polymorphisms of the MTHFR gene linked with altered enzymatic activity and hyperhomocysteinemia are the $677 \mathrm{C} / \mathrm{T}$ (Ala to Val at codon 222) (46) and 1298A/C (Glu to Ala at codon 428) (47) single nucleotide polymorphisms. However, the polymorphic allele 1298C does not modify MTHFR enzymatic activity in the same way as it does in the $677 \mathrm{~T}$ allele. The MTHFR $677 \mathrm{C}>\mathrm{T}$ polymorphism consists of a substitution of cytosine for thymine at position 677 , resulting in the exchange of alanine for valine at residue 222 .

A French study examined the relationship between the MTHFR gene polymorphisms 677C/T and 1298A/C and clinical response to the FOLFOX protocol. The authors observed response rates of 53,63,80 and 37\% in CRC patients harboring one, two, three and none of the favorable MTHFR alleles, respectively. These data indicate that the MTHFR germinal polymorphisms may represent predictive markers of response to FOLFOX therapy. Moreover, for the first time, this study demonstrated that the response rate to FOLFOX chemotherapy proportionally increases with the number of favorable MTHFR alleles (48).

Another study by Castillo-Fernández et al focused on only the MTHFR $677 \mathrm{C}>\mathrm{T}$ polymorphism in metastatic colorectal cancer patients treated with 5FU and folinic acid (FA), and showed that MTHFR $677 \mathrm{C}>\mathrm{T}$ polymorphism is associated with longer time to progression and overall survival in these patients treated with 5-FU/FA. These findings are in agreement with the results of the French group (49).

VEGF C936T polymorphism and bevacizumab. VEGF is essential in the regulation of physiological and pathological angiogenesis, as it is also produced by cancer cells in a hypoxic state. Polymorphisms of the VEGF gene have been identified and correlated with variations in VEGF protein production. Our unpublished data show that the VEGF C936T polymorphism is associated with increased plasma levels of VEGF in cancer patients with deep vein thrombosis (DVT). Additionally, our data show that this polymorphism may be a predictive indicator of DVT in colorectal cancer patients treated with bevacizumab. However, further studies are required to confirm our preliminary observations.

Other genetic alterations. As described above, anti-EGFR therapy is useful for the treatment of colorectal cancer. Whether patients will benefit from this treatment is based on the K-Ras mutation status of the tumor DNA. However, other predictive markers of response in addition to K-Ras mutations have been proposed. For example, an increased response rate to antiEGFR antibodies has been observed in colon cancer patients with an elevated number of copies of EGFR $(50,51)$. Similarly, there have been reports implicating mutations of the PI3K gene in the response to anti-EGFR agents $(52,53)$; however, the data generated by such studies are conflicting (54).

\section{Conclusions}

The present review discusses the growing importance of tailored therapy for colorectal cancer patients. It is becoming increasingly clear that further studies are required on all the possible genetic variables (e.g., polymorphisms and mutations) that affect individual response to specific chemotherapeutic agents. The evidence indicates that some polymorphisms enhance the activity of certain drugs, while others greatly increase their toxicity. 
The study of the genetic 'habitus' of cancer patients therefore appears to be crucial in order to develop improved therapeutic regimens and to combat chemoresistance. The optimal chemotherapy regimen may be not identical for all patients, thus the development of tailored therapies should be the focus of future studies on anti-cancer therapy.

\section{References}

1. Burt RW, Bishop DT, Lynch HT, Rozen P and Winawer SJ: Risk and surveillance of individuals with heritable factors for colorectal cancer. WHO Collaborating Centre for the Prevention of Colorectal Cancer. Bull World Health Organ 68: 655-665, 1990.

2. Mandel JS, Bond JH, Church TR, Snover DC, Bradley GM, Schuman LM and Ederer F: Reducing mortality from colorectal cancer by screening for fecal occult blood. Minnesota Colon Cancer Control Study. N Engl J Med 328: 1365-1371, 1993.

3. Wingo PA, Tong T and Bolden S: Cancer statistics, 1995. CA Cancer J Clin 45: 8-30, 1995.

4. Tol J and Punt CJ: Monoclonal antibodies in the treatment of metastatic colorectal cancer: a review. Clin Ther Mar 32: 437-453, 2010

5. Duff SE, Jeziorska M, Rosa DD, et al: Vascular endothelial growth factors and receptors in colorectal cancer: implications for anti-angiogenic therapy. Eur J Cancer 42: 112-117, 2006.

6. Dvorak HF: Vascular permeability factor/vascular endothelial growth factor: a critical cytokine in tumor angiogenesis and a potential target for diagnosis and therapy. J Clin Oncol 20 : 4368-4380, 2002.

7. Des Guetz G, Uzzan B, Nicolas P, et al: Microvessel density and VEGF expression are prognostic factors in colorectal cancer Meta-analysis of the literature. Br J Cancer 94: 1823-1832, 2006.

8. Rajaganeshan R, Prasad R, Guillou PJ, et al: The influence of invasive growth pattern and microvessel density on prognosis in colorectal cancer and colorectal liver metastases. Br J Cancer 96: $1112-1117,2007$

9. Jubb AM, Hurwitz HI, Bai W, et al: Impact of vascular endothelial growth factor-A expression, thrombospondin-2 expression, and microvessel density on the treatment effect of bevacizumab in metastatic colorectal cancer. J Clin Oncol 24: 217-227, 2006.

10. Scaltriti $\mathrm{M}$ and Baselga $\mathrm{J}$ : The epidermal growth factor receptor pathway: a model for targeted therapy. Clin Cancer Res 12: 5268-5272, 2006.

11. Goldstein NS and Armin M: Epidermal growth factor receptor immunohistochemical reactivity in patients with American Joint Committee on Cancer Stage IV colon adenocarcinoma: implications for a standardized scoring system. Cancer 92: 1331-1346, 2001.

12. Spano JP, Lagorce C, Atlan D, et al: Impact of EGFR expression on colorectal cancer patient prognosis and survival. Ann Oncol 16: 102-108, 2005.

13. Zlobec I, Vuong T, Hayashi S, et al: A simple and reproducible scoring system for EGFR in colorectal cancer: application to prognosis and prediction of response to preoperative brachytherapy. Br J Cancer 96: 793-800, 2007.

14. De Roock W, Piessevaux H, De Schutter J, et al: KRAS wild-type state predicts survival and is associated to early radiological response in metastatic colorectal cancer treated with cetuximab. Ann Oncol 19: 508-515, 2008.

15. Di Fiore F, Blanchard F, Charbonnier F, et al: Clinical relevance of KRAS mutation detection in metastatic colorectal cancer treated by Cetuximab plus chemotherapy. $\mathrm{Br} \mathrm{J}$ Cancer 96 : 1166-1169, 2007.

16. Lièvre A, Bachet JB, Boige V, et al: KRAS mutations as an independent prognostic factor in patients with advanced colorectal cancer treated with cetuximab. J Clin Oncol 26: 374-379, 2008.

17. Garm Spindler KL, Pallisgaard N, Rasmussen AA, et al: The importance of KRAS mutations and EGF61 A $>$ G polymorphism to the effect of cetuximab and irinotecan in metastatic colorectal cancer. Ann Oncol 20: 879-884, 2009.

18. Loupakis F, Pollina L, Stasi I, et al: PTEN expression and KRAS mutations on primary tumors and metastases in the prediction of benefit from cetuximab plus irinotecan for patients with metastatic colorectal cancer. J Clin Oncol 27: 2622-2629, 2009.

19. Andreyev HJ, Norman AR, Cunningham D, et al: Kirsten ras mutations in patients with colorectal cancer: the multicenter 'RASCAL' study. J Natl Cancer Inst 90: 675-684, 1998.
20. Benvenuti S, Sartore-Bianchi A, Di Nicolantonio F, et al: Oncogenic activation of the RAS/RAF signaling pathway impairs the response of metastatic colorectal cancers to antiepidermal growth factor receptor antibody therapies. Cancer Res 67: 2643-2648, 2007.

21. European Medicines Agency. Committee for Medicinal Products for Human Use Post-authorisation Summary of Positive Opinion for Erbitux. http://www.emea.europa.eu/pdfs/human/opinion/ Erbitux_28040208en. pdf. Accessed December 1, 2009.

22. Tol J, Nagtegaal ID and Punt CJ: BRAF mutation in metastatic colorectal cancer. N Engl J Med 361: 98-99, 2009.

23. Bibeau F, Lopez-Crapez E, Di Fiore F, et al: Impact of Fc $\gamma$ RIIaFc $\gamma$ RIIIa polymorphisms and KRAS mutations on the clinical outcome of patients with metastatic colorectal cancer treated with cetuximab plus irinotecan. J Clin Oncol 27: 1122-1129, 2009.

24. Duff SE, Jeziorska M, Rosa DD, et al: Vascular endothelial growth factors and receptors in colorectal cancer: implications for anti-angiogenic therapy. Eur J Cancer 42: 112-117, 2006.

25. Antonacopoulou AG, Tsamandas AC, Petsas T, et al: EGFR, HER-2 and COX-2 levels in colorectal cancer. Histopathology 53: 698-706, 2008

26. Niu G, Wright KL, Huang M, et al: Constitutive Stat3 activity upregulates VEGF expression and tumor angiogenesis. Oncogene 21: 2000-2008, 2002.

27. Jung YD, Mansfield PF, Akagi M, et al: Effects of combination antivascular endothelial growth factor receptor and anti-epidermal growth factor receptor therapies on the growth of gastric cancer in a nude mouse model. Eur J Cancer 38: 1133-1140, 2002.

28. Shaheen RM, Ahmad SA, Liu W, et al: Inhibited growth of colon cancer carcinomatosis by antibodies to vascular endothelial and epidermal growth factor receptors. Br J Cancer 85: 584-589, 2001.

29. Tonra JR, Deevi DS, Corcoran E, et al: Synergistic antitumor effects of combined epidermal growth factor receptor and vascular endothelial growth factor receptor-2 targeted therapy. Clin Cancer Res 12: 2197-2207, 2006.

30. Ciardiello F, Bianco R, Damiano V, et al: Antiangiogenic and antitumor activity of anti-epidermal growth factor receptor $\mathrm{C} 225$ monoclonal antibody in combination with vascular endothelial growth factor antisense oligonucleotide in human GEO colon cancer cells. Clin Cancer Res 6: 3739-3747, 2000.

31. Tol J and Punt CJA: Monoclonal antibodies in the treatment of metastatic colorectal cancer: a review. Clin Ther 32: 437-453, 2010.

32. Zhang W, Gordon M, Schultheis AM, et al: FCGR2A and FCGR3A polymorphisms associated with clinical outcome of epidermal growth factor receptor expressing metastatic colorectal cancer patients treated with single-agent cetuximab. J Clin Oncol 25: 3712-3718, 2007.

33. Weinshilboum R: Inheritance and drug response. N Engl J Med 348: 529-537, 2003.

34. Evans WE and McLeod H: Pharmacogenomics: drug disposition, drug targets, and side effects. N Engl J Med 348: 538-549, 2003.

35. Goldstein DB: Pharmacogenetics in the laboratory and the clinic. N Engl J Med 348: 553-556, 2003.

36. Chen ZP, Malapetsa A, Monks A, et al: Nucleotide excision repair protein levels vis-a-vis anticancer drug resistance in 60 human tumor cell lines. Ai Zheng 21: 233-239, 2002.

37. Park DJ, Stoehlmacher J, Zhang W, et al: A Xeroderma pigmentosum group $\mathrm{D}$ gene polymorphism predicts clinical outcome to platinum-based chemotherapy in patients with advanced colorectal cancer. Cancer Res 61: 8654-8658, 2001

38. Toffoli G, Cecchin E, Corona G, et al: The role of UGT1A1*28 polymorphism in the pharmacodynamics and pharmacokinetics of irinotecan in patients with metastatic colorectal cancer. J Clin Oncol 24: 3061-3068, 2006

39. Stoehlmacher J, Park DJ, Zhang W, et al: Association between glutathione S-transferase P1, T1, and M1 genetic polymorphism and survival of patients with metastatic colorectal cancer. J Natl Cancer Inst 94: 936-942, 2002.

40. Stoehlmacher J, Ghaderi V, Iobal S, et al: A polymorphism of the $\mathrm{XRCC} 1$ gene predicts for response to platinum based treatment in advanced colorectal cancer. Anticancer Res 21: 3075-3079, 2001.

41. Hurwitz H, Fehrenbacher L, Novotny W, et al: Bevacizumab plus irinotecan, fluorouracil, and leucovorin for metastatic colorectal cancer. N Engl J Med 350: 2335-2342, 2004. 
42. Libra M, Navolanic PM, Talamini R, Cecchin E, Sartor F, Tumolo S, Masier S, Travali S, Boiocchi M and Toffoli G: Thymidylate synthetase mRNA levels are increased in liver metastases of colorectal cancer patients resistant to fluoropyrimidine-based chemotherapy. BMC Cancer 4: 11, 2004.

43. Horie N, Aiba H, Oguro K, Hojo H and Takeishi K: Functional analysis and DNA polymorphism of the tandemly repeated sequences in the 5'-terminal regulatory region of the human gene for thymidylate synthase. Cell Struct Funct 20: 191-197, 1995.

44. Pàez D, Parè L, Altès $\mathrm{A}$, et al: Thymidylate synthase germline polymorphisms in rectal cancer patients treated with neoadjuvant chemoradiotherapy based on 5-fluorouracil. J Cancer Res Clin Oncol 136: 1681-1689, 2010.

45. Park JH, Kim NS, Park JY, et al: MGMT 2535G $>$ T polymorphism is associated with prognosis for patients with metastatic colorectal cancer treated with oxaliplatin-based chemotherapy. J Cancer Res Clin Oncol 136: 1135-1142, 2010.

46. Rozen R: Molecular genetics of methylenetetrahydrofolate reductase deficiency. J Inherti Metab Dis 19: 589-594, 1996.

47. Weisberg I, Tran P, Christensen B, Sibani S and Rozen R: A second genetic polymorphism in methylenetetrahydrofolate reductase (MTHFR) associated with decreased enzyme activity. Mol Genet Metab 64: 169-172, 1998

48. Etienne-Grimaldi MC, Milano G, Maindrault-Goebel F, et al: Methylenetetrahydrofolate reductase (MTHFR) gene polymorphisms and FOLFOX response in colorectal cancer patients. Br J Clin Pharmacol 69: 58-66, 2010.
49. Castillo-Fernándeza O, Santibáñezb M, Bauzab A, et al: Methylenetetrahydrofolate reductase polymorphism $(677 \mathrm{C}>\mathrm{T})$ predicts long time to progression in metastatic colon cancer treated with 5-fluorouracil and folinic acid. Arch Med Res 41: 430-435, 2010.

50. Moroni M, Veronese S, Benvenuti S, et al: Gene copy number for epidermal growth factor receptor (EGFR) and clinical response to antiEGFR treatment in colorectal cancer: a cohort study. Lancet Oncol 6: 279-286, 2005.

51. Sartore-Bianchi A, Moroni M, Veronese S, et al: Epidermal growth factor receptor gene copy number and clinical outcome of metastatic colorectal cancer treated with panitumumab. J Clin Oncol 25: 3238-3245, 2007

52. Sartore-Bianchi A, Martini M, Molinari F, et al: PIK3CA mutations in colorectal cancer are associated with clinical resistance to EGFR-targeted monoclonal antibodies. Cancer Res 69: 1851-1857, 2009.

53. Prenen H, De Schutter J, Jacobs B, et al: PIK3CA mutations are not a major determinant of resistance to the epidermal growth factor receptor inhibitor cetuximab in metastatic colorectal cancer. Clin Cancer Res 15: 3184-3188, 2009.

54. Di Nicolantonio F, Martini M, Molinari F, et al: Wild-type $\mathrm{BRAF}$ is required for response to panitumumab or cetuximab in metastatic colorectal cancer. J Clin Oncol 26: 5705-5712, 2008. 